CARVALHO, W.; FONSECA, M.E.N.; SILVA, H.R.; BOITEUX, L.S.; GIORDANO, L.B. Estimativa indireta de teores de licopeno em frutos de genótipos de tomateiro via análise colorimétrica. Horticultura Brasileira, Brasília, v.232 n.3, p.819-825, jul-set 2005.

\title{
Estimativa indireta de teores de licopeno em frutos de genótipos de tomateiro via análise colorimétrica
}

\author{
Wesley Carvalho, ${ }^{1,2,3}$; Maria Esther de N. Fonseca ${ }^{1}$; Henoque R. da Silva ${ }^{1}$; Leonardo S. Boiteux ${ }^{1,4}$; Leonar- \\ do de B. Giordano ${ }^{1,4}$ \\ ${ }^{1}$ Embrapa Hortaliças, C. Postal 218, 70359-970 Brasília-DF; ${ }^{2}$ UnB, Instituto de Química, 70910-900 Brasília-DF; ${ }^{3}$ Bolsista do Programa \\ Institucional de Iniciação Científica - PIBIC (CNPq); ${ }^{4}$ Bolsista CNPq; E-mail: giordano@cnph.embrapa.br
}

\section{RESUMO}

O pigmento vermelho licopeno $\left(\mathrm{C}_{40} \mathrm{H}_{56}\right)$ é um carotenóide que acumula em frutos, flores e raízes de algumas espécies vegetais, incluindo, hortaliças e fruteiras. A principal fonte de licopeno na dieta humana é o fruto do tomateiro (Lycopersicon esculentum Mill.) e seus derivados. A estrutura química singular do licopeno confere marcante ação antioxidante, contribuindo na prevenção de doenças degenerativas, cardiovasculares e de certos tipos de câncer. Além do fator nutricional, a concentração do licopeno no tomate está relacionada com uma melhor percepção visual dos produtos, existindo, portanto, uma forte demanda para aumentar os teores deste pigmento em frutos das cultivares tanto para consumo in natura quanto para processamento industrial. O desenvolvimento de cultivares com teores mais elevados de fatores nutricionais, incluindo licopeno, depende da disponibilidade de métodos de seleção simples e precisos. O objetivo deste trabalho foi estimar os níveis de correlação entre os teores de licopeno determinados via espectrofotometria com os valores dos componentes de cromaticidade ( $\left.\mathrm{L}^{*}, \mathrm{a}^{*} \mathrm{e} \mathrm{b}^{*}\right)$ obtidos via análise colorimétrica. Foram utilizadas para a análise colorimétrica três formas de amostragem: (1) leitura da superfície externa da parede dos frutos; (2) leitura da superfície interna da parede dos frutos e (3) leitura da cor da polpa homogeneizada. Para o componente L* (grau relativo de brilho), obteve-se valores de $\mathrm{R}^{2}$ entre 0,63 a 0,82 , sendo que a leitura da polpa homogeneizada apresentou a maior correlação. Os valores do descritor cromático $\mathrm{a}^{*}$ (+a*: grau da cor vermelha; - $\mathrm{a}^{*}$ : grau da cor verde) apresentaram valores de $\mathrm{R}^{2}$ variando de 0,79 a 0,82 . Os valores do descritor cromático $b^{*}\left(+b^{*}\right.$ : grau da cor amarela; -b*: grau da cor azul) mostraram correlações mais baixas, porém ainda significativas $\left(\mathrm{R}^{2}\right.$ entre 0,27 e 0,46$)$. Os valores mais expressivos de $\mathrm{R}^{2}$ (entre 0,86 e 0,91 ) foram obtidos quando a concentração de licopeno foi correlacionada com os resultados das relações $a^{*} / b^{*}$ e $\left(a^{*} / b^{*}\right)^{2}$, sendo que os valores de cromaticidade da polpa homogeneizada apresentaram novamente as mais elevadas correlações. Estes resultados confirmam que é possível estimar indiretamente e com relativa precisão, o teor de licopeno em frutos do tomateiro a partir de valores de cromaticidade. Esta metodologia pode ser empregada para seleção de genótipos com maiores teores de licopeno, de maneira mais simplificada, evitando o dispêndio e os problemas de descarte dos solventes orgânicos utilizados nos métodos espectrofotométricos.

Palavras-chave: Lycopersicon esculentum, colorímetro, pigmentos, carotenóides.

\section{ABSTRACT}

Indirect estimation of lycopene concentration in fruits of tomato genotypes via chromaticity values

Lycopene $\left(\mathrm{C}_{40} \mathrm{H}_{56}\right)$ is a red carotenoid pigment, which naturally accumulates in flowers, fruits and roots of some vegetable and fruit crops. The unique properties of the lycopene molecule make this pigment one of the most powerful antioxidants available. Tomato (Lycopersicon esculentum Mill.) fruits and processed tomato products are the main sources of lycopene in the human diet. There is a growing amount of experimental data indicating that lycopene intake is associated with decreased risk of many types of degenerative and cardiovascular diseases as well as cancers in humans. In addition, lycopene concentration is associated with improved visual perception of tomato products. Therefore, there is a strong demand to increase the total amount of lycopene in tomato cultivars for either fresh market or processing. The development of cultivars for improved lycopene content is dependent upon the availability of simple yet accurate methodologies to quantify this carotene in tomato fruits and processed tomato products. The main objective of this work was to estimate the degree of correlation between the total lycopene content in tomato fruits measured spectrophotometrically and the chromaticity values $\left(\mathrm{L}^{*}, \mathrm{a}^{*}\right.$ and $\left.\mathrm{b}^{*}\right)$ obtained using a tristimulus colorimeter. Colorimetric readings were taken using three sampling strategies: (1) external fruit wall; (2) internal fruit wall and (3) the whole homogenized fruit pulp. For the achromatic component $\mathrm{L}^{*}$ (relative darkness or lightness), the obtained $\mathrm{R}^{2}$ values were within 0.63 and 0.82 , with the whole homogenized fruit pulp providing the highest correlation. For the chromatic component a* (green to red) the correlation $\mathrm{R}^{2}$ values ranged from 0.79 to 0.82 . For the chromatic component $\mathrm{b}^{*}$ (blue to yellow) lower yet significant values were observed ( $\mathrm{R}^{2}$ between 0.27 and 0.46$)$. However, it was observed that lycopene content was best correlated with the mathematical relationships $\mathrm{a}^{*} / \mathrm{b}^{*}\left(\mathrm{R}^{2}\right.$ range from 0.88 to 0.90$)$ and $\left(\mathrm{a}^{*} / \mathrm{b}^{*}\right)^{2}\left(\mathrm{R}^{2}\right.$ range from 0.86 to 0.91 ), with these correlation values being consistently higher when the chromaticity readings were taken in whole homogenized fruit pulp. These results confirmed the feasibility of obtaining precise indirect estimation of lycopene content from chromaticity readings. The methodology described here could be useful for large scale selection of tomato lines with improved levels of lycopene without of high costs and of residue disposal problems associated with the employment of organic solvents in the standard spectrophotometric methods.

Keywords: Lycopersicon esculentum, colorimeter, pigments, carotenoids, lycopene.

(Recebido para publicação em 4 de novembro de 2004 e

aceito em 6 de junho de 2005)

$\mathrm{O}$ pigmento licopeno $\left(\mathrm{C}_{40} \mathrm{H}_{56}\right)$ pertence ao subgrupo dos carotenóides não oxigenados, sendo caracterizado por uma estrutura acíclica e simétrica con- tendo 11 ligações duplas conjugadas (RAO, 2002). Devido a sua estrutura química, o licopeno figura como um dos melhores supressores biológicos de ra- dicais livres, especialmente aqueles derivados do oxigênio. Entre uma série de carotenóides avaliados, o licopeno mostrou-se como um dos mais eficientes 
antioxidantes, podendo doar elétrons para neutralizar as moléculas de oxigênio singleto e outras moléculas oxidantes antes que elas prejudiquem as células (RAO et al., 1998; RAO; AGAWAL, 2000). Estudos clínicos e epidemiológicos têm confirmado que dietas ricas em licopeno estão associadas com a redução do risco de desenvolvimento de câncer de próstata e ovário bem como a uma menor incidência de doenças degenerativas crônicas e cardiovasculares (NGUYEN; SCHWARTZ, 1999; CRAMER et al., 2001; RAO, 2002). A principal fonte de licopeno na dieta humana é o fruto do tomate (Lycopersicon esculentum Mill.) e seus derivados tais como sucos, sopas, molhos e 'catchups'. É interessante salientar que alguns trabalhos mais recentes indicam que a ingestão de licopeno presente no fruto do tomate é mais eficiente na prevenção de certos tipos de câncer do que a administração do licopeno purificado via cápsulas (BOILEAU et al., 2003).

A produção de tomate no Brasil é de aproximadamente 3 milhões de toneladas/ano sendo $65 \%$ destinado ao consumo in natura e $35 \%$ para processamento industrial. Isto equivale dizer que são produzidas anualmente cerca de 1.500 t da molécula de licopeno na safra brasileira (assumindo teores médios de licopeno de $50 \mu \mathrm{g} / \mathrm{g}$ ). Atualmente, a capacidade das indústrias de processamento instaladas no Brasil é de aproximadamente $15.185 \mathrm{t} /$ dia de pasta de tomate e de $1.495 \mathrm{t} /$ dia de tomate em cubos. Na agroindústria, existe uma demanda por itens processados de maior valor agregado que combinem aroma, sabor e elevada pigmentação vermelha de polpa (conferida pela presença de licopeno). A combinação destes fatores é essencial para alavancar os produtos derivados de tomate aos níveis de qualidade necessários para atingir nichos de elevado padrão de exigência, tanto no mercado doméstico quanto no exterior. Além do fator nutricional, tem sido demonstrado que teores de pigmentos carotenóides tais como o licopeno estão fortemente relacionados com uma melhor percepção visual dos produtos. Neste contexto, existe uma demanda da parte de consumidores, varejistas e das agroindústrias processadoras de polpa de tomate no sentido de melhorar o teor de licopeno dos frutos das cultivares atualmente comercializadas, tanto para consumo in natura quanto para processamento. Desta forma, cabe aos programas de melhoramento genético a tarefa de diversificar o panorama varietal do tomateiro disponibilizando aos consumidores materiais que combinem fatores nutricionais, principalmente o licopeno, com sabor e aroma. Para tal, torna-se necessária a disponibilidade de métodos simples, práticos e de baixo custo a fim de acelerar o processo de identificação e seleção de linhagens com maiores teores de licopeno dentro dos programas de melhoramento genético desta hortaliça.

A metodologia padrão empregada na determinação dos teores de licopeno em tomate tem sido essencialmente a mesma utilizada em outras espécies de hortaliças e frutas (LIME et al., 1957; UMIEL; GABELMAN, 1971; MORETTI et al., 1998; ARIAS et al., 2000; RODRIGUEZ-AMAYA, 2001). Nestas metodologias, a concentração de carotenóides é determinada por meio de análise espectrofotométrica ou via "High Performance Liquid Chromatography" (HPLC). Para o caso de frutos maduros de cultivares tradicionais de tomateiro, as análises espectrofotométricas empregando carotenóides totais tem sido utilizada como uma estimativa dos teores de licopeno uma vez que este é o carotenóide predominante. No procedimento utilizando espectrofotômetro, os carotenóides totais são inicialmente extraídos dos frutos utilizando-se o solvente orgânico acetona e transferidos para éter de petróleo com posterior leitura no comprimento de onda de 470 $\mathrm{nm}$.

Os métodos espectrofotométricos descritos na literatura são bastante precisos, entretanto muito trabalhosos, sendo necessária a utilização de uma quantidade excessiva de reagentes, além de demandar muito tempo. Devido à conveniência e maior facilidade no uso de medidas de cor, vários estudos têm investigado a intensidade da correlação entre valores de cromaticidade e o teor de pigmentos de diferentes hortaliças. Em tomate, estes estudos têm demons- trado uma boa correlação entre cor de fruto e teor de licopeno (D'SOUZA et al., 1992; ARIAS et al., 2000). Identificação de elevados níveis de correlação entre leituras colorimétricas e teores de licopeno poderão permitir o estabelecimento de um método mais prático servindo como uma ferramenta no processo de seleção de genótipos nos programas de melhoramento genético do tomateiro. Desta forma, neste trabalho investigou-se a intensidade de correlação entre a concentração de licopeno em frutos do tomateiro com a medida de sua cromaticidade utilizando três formas de amostragem: (1) leitura da cor de polpa homogeneizada; (2) leitura da superfície externa do fruto e (3) leitura da parte interna da parede dos frutos.

\section{MATERIAL E MÉTODOS}

Os híbridos $\mathrm{F}_{1}$ de tomate 'Heinz 9492', 'RPT 1095' e 'UG 096', foram cultivados na Fazenda Agriter, em Cristalina $(\mathrm{GO})$ e os frutos foram colhidos em setembro de 2003. Estes frutos foram lavados, secados e classificados em seis estádios de maturação (The

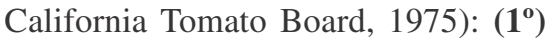
Verde-maduro: $100 \%$ da superfície possuindo coloração verde, com tonalidade que varia de verde-clara a escura; $\left(\mathbf{2}^{\mathbf{o}}\right)$ Verde-rosado: entre $0 \%$ a $10 \%$ da superfície do fruto possuindo coloração avermelhada ou amarelada; (3') Rosaesverdeado: entre $10 \%$ e $30 \%$ da superfície do fruto com coloração avermelhada, rósea ou amarelada; $\left(4^{\circ}\right)$ Róseo: entre $30 \%$ e $60 \%$ da superfície do fruto com coloração avermelhada ou rósea; $\left(\mathbf{5}^{\mathbf{}}\right)$ Vermelho-claro: entre $60 \%$ e $90 \%$ da superfície do fruto, não mais do que $90 \%$ da soma de todas as áreas superficiais, possuindo coloração róseoavermelhada ou vermelha e $\left(\mathbf{6}^{\mathbf{0}}\right)$ Vermelho: mais de $90 \%$ da superfície do fruto, na soma de todas as áreas superficiais, possuindo coloração vermelho-intensa.

Foram amostrados quatro frutos em cada estádio. A cor foi medida com colorímetro "Tristimulus" (Minolta Modelo CR-200b), com 8 mm de diâmetro da área de medida do aparelho e com iluminação difusa (iluminante D65, ângulo de visão $0^{\circ}$ e espaço cromático 
CIELAB). O aparelho foi calibrado no sistema $\mathrm{L}^{*}, \mathrm{a}^{*}, \mathrm{~b}^{*}$ com uma placa branca padrão de cerâmica $\left(\mathrm{L}^{*}=84,2 ; \mathrm{a}^{*}=\right.$ 10,$\left.1 ; b^{*}=14,6\right)$. Foram feitas quatro leituras na região mediana dos frutos no sentido longitudinal: duas leituras nas região externa e duas leituras na região interna da parede dos frutos. Também foram feitas leituras da coloração do fruto homogeneizado em um Mixer Vertical SB30. A polpa foi vertida completando-se o volume de uma placa de Petri com nove centímetros de diâmetro, procedendo-se a leitura da cor na superfície da polpa. Depois de serem feitas as medidas, a polpa do fruto foi guardada em congelador $\left(-30^{\circ} \mathrm{C}\right.$, no escuro) protegida por papel alumínio. A concentração de licopeno nos frutos foi obtida por análise espectrofotométrica. Após o descongelamento da polpa, foram tomadas amostras de 5,0 g. Em cada amostra adicionou-se $40 \mathrm{ml}$ de acetona procedendo-se, em seguida, uma agitação da mistura por 1 hora utilizando-se um agitador Multi Shaker MMS a 200 rpm. Em seguida, procedeu-se a filtragem a vácuo com o auxílio de um Kitassato envolto em papel alumínio para evitar a foto-oxidação dos pigmentos. Cada amostra foi lavada com acetona por mais três vezes objetivando a total extração dos pigmentos. Ao funil de separação foram adicionados $45 \mathrm{ml}$ de éter de petróleo. Os pigmentos foram então transferidos, em pequenas frações, seguidas de água destilada, para o funil de separação, descartando-se a fase inferior. As amostras foram lavadas por mais quatro vezes para remoção total da acetona. A solução dos pigmentos em éter de petróleo foi transferida para um balão volumétrico completando-se o volume para $100 \mathrm{ml}$ com éter de petróleo. A leitura no espectrofotômetro (Micronal B495) foi feita no comprimento de onda de $470 \mathrm{~nm}$ (RODRIGUEZ-AMAYA, 2001).

O teor de licopeno foi obtido pela fórmula:

$$
\mu g / g=\frac{(A \times V \times 1.000 .000)}{\left(A_{1 \mathrm{~cm}}^{1 \%} \times \mathrm{M} \times 100\right)}
$$

Onde A = absorvância da solução no comprimento de onda de $470 \mathrm{~nm}, \mathbf{V}=$ volume final da solução, $A_{1 \mathrm{~cm}}^{1 \%}$ é o coeficiente de extinção ou coeficiente de absortividade molar de um pigmento em um determinado solvente específico e $\mathbf{M}=$ massa da amostra tomada para a análise. Para o licopeno em éter de petróleo o valor do coeficiente de extinção é 3450 (RODRIGUEZ-AMAYA, 2001). Os dados foram analisados via "General Linear Models Procedure" e "Regression Analysis" dentro do aplicativo computacional SAS (SAS INSTITUTE, 1982).

\section{RESULTADOS E DISCUSSÃO}

As análises espectrofotométricas dos teores de licopeno [expressos em $\mu \mathrm{g} / \mathrm{g}$ $\pm \mathrm{s}(\hat{\mathrm{m}})]$ na polpa dos frutos dos três híbridos avaliados apresentaram os seguintes valores: Híbrido 'Heinz 9492': $4,93( \pm 1,53) ; 11,16( \pm 3,80) ; 26,09$ $( \pm 7,07) ; 57,39( \pm 7,96) ; 73,04( \pm 14,64)$; 101,88 ( $\pm 8,24)$; Híbrido 'RPT 1095': $3,91( \pm 1,52) ; 7,34( \pm 1,86) ; 16,61$ $( \pm 4,50) ; 33,23( \pm 18,86) ; 68,40( \pm 10,04)$; 105,94 $( \pm 23,26)$ e Híbrido 'UG 096': $1,59( \pm 0,55) ; 24,05( \pm 4,50) ; 32,17$ $( \pm 1,46) ; 56,66( \pm 6,61), 62,75( \pm 6,10)$, $82,32( \pm 15,17)$. Estes seis valores correspondem, respectivamente, para cada híbrido, aos obtidos de frutos nos estádios verde-maduro, verde-rosado, rosa-esverdeado, róseo, vermelho claro e vermelho. O híbrido 'RPT 1095' foi o único que apresentou frutos com amadurecimento heterogêneo no segundo, terceiro e quarto estádios de maturação.

Os valores do componente acromático L* (grau de brilho) decresceram à medida que os frutos passaram do estádio de verde-maduro para vermelho nas leituras feitas na parede externa, parede interna e na polpa homogeneizada dos frutos dos híbridos avaliados (Tabelas 1, 2 e 3). Estes resultados estão de acordo com observações prévias que indicam que o valor de L* decai, com o aparecimento da cor vermelha, à medida que os frutos amadurecem representando a perda de brilho dos frutos devido à síntese de carotenóides e diminuição da coloração verde (LÓPEZ CAMELO; GÓMEZ, 2004). É interessante observar que os valores de $\mathrm{L}^{*}$ foram maiores na polpa homogeneizada quando comparadas com as leituras feitas na paredes interna e externa dos frutos (Tabelas 1, 2 e 3). Este fato reflete, provavelmente, a presença do tecido placentário na polpa homogeneizada uma vez que $\mathrm{L}^{*}$ é um descritor acromático.

Os valores do componente cromático a* ( $+\mathrm{a}^{*}$ : grau da cor vermelha do fruto; -a*: grau da cor verde) nas três formas de amostragem e nos três híbridos aumentaram à medida que os frutos passaram de verde-maduro (valores negativos) para vermelho (valores positivos) como conseqüência da síntese de licopeno e redução da clorofila. Os valores do componente cromático $b^{*}\left(+b^{*}\right.$ : grau da cor amarela; -b*: grau da cor azul) revelaram tendência de acréscimo até o terceiro estádio de maturação, com acentuado decréscimo nos estádios posteriores, correspondendo a uma acentuada síntese de licopeno (cor vermelha) e beta-caroteno (cor laranja), que mascaram os pigmentos de coloração amarela (por exemplo zeta-caroteno) (LOPEZCAMELO; GÓMEZ, 2004).

As relações $a^{*} / b^{*}$ e $\left(a^{*} / b^{*}\right)^{2}$ têm sido normalmente usadas como índice de maturação dos frutos e apresentam elevada correlação com os diferentes estádios de maturação (LOPEZ-CAMELO; GÓMEZ, 2004). No presente trabalho, foi calculada a regressão linear entre os estádios de maturação (teores de licopeno) e os valores de $\mathrm{a}^{*} / \mathrm{b}^{*}$ e $\left(\mathrm{a}^{*} / \mathrm{b}^{*}\right)^{2}$ (Figura 1). As análises de regressão linear simples entre os teores de licopeno e a cor de polpa, e das paredes externa e interna dos frutos encontram-se na Tabela 4. Para o parâmetro L*, nas leituras feitas na parte externa e interna das paredes dos frutos, obteve-se moderada correlação com os valores de licopeno $\left(R^{2}=0,67\right.$ e 0,63 , respectivamente), sendo que os valores de $\mathrm{L}^{*}$ para a polpa homogeneizada foi a que apresentou maior correlação com os teores de licopeno $\left(\mathrm{R}^{2}=0,82\right)$. Alguns estudos têm mostrado elevada correlação entre o valor de $L^{*}$ na superfície externa e interna do fruto e o teor de licopeno em tomate, variando de $\mathrm{R}^{2}=0,82$ (D'SOUZA et al., 1992) a 0,84 (ARIAS et al., 2000).

Os valores de $\mathrm{a}^{*}$ obtidos das leituras de cromaticidade realizadas nas paredes 
Tabela 1. Valores dos componentes colorimétricos L", a* e b* de frutos do híbrido 'Heinz 9492' obtidos de diferentes estádios de maturação em três tipos de amostragem. Brasília, Embrapa Hortaliças, 2004.

\begin{tabular}{|c|c|c|c|c|c|c|}
\hline $\begin{array}{l}\text { Método de } \\
\text { amostragem }\end{array}$ & $\begin{array}{l}\text { Estádios de } \\
\text { maturação }\end{array}$ & $\mathrm{L}^{*}$ & $a^{*}$ & $\mathbf{b}^{*}$ & $a^{*} / b^{*}$ & $\left(a^{*} / b^{*}\right)^{2}$ \\
\hline \multirow{6}{*}{$\begin{array}{l}\text { Parede interna } \\
\text { do fruto }\end{array}$} & Verde-maduro & $53,95 \pm 1,9$ & $-12,58 \pm 1,8$ & $28,49 \pm 3,4$ & $-0,44 \pm \quad 0,0$ & $0,19 \pm 0,0$ \\
\hline & Verde-rosado & $48,71 \pm 1,5$ & $-3,05 \pm 0,6$ & $26,29 \pm 2,9$ & $-0,12 \pm 0,0$ & $0,01 \pm 0,0$ \\
\hline & Rosa-esverdeado & $46,11 \pm 5,2$ & $18,09 \pm 7,9$ & $29,13 \pm 4,6$ & $0,37 \pm 0,3$ & $0,20 \pm 0,2$ \\
\hline & Róseo & $36,75 \pm 3,6$ & $26,75 \pm 6,1$ & $20,36 \pm 2,9$ & $1,32 \pm 0,3$ & $1,82 \pm \quad 0,7$ \\
\hline & Vermelho-claro & $35,11 \pm 3,5$ & $26,91 \pm 1,5$ & $18,04 \pm 1,5$ & $1,49 \pm 0,1$ & $2,24 \pm 0,2$ \\
\hline & Vermelho & $33,49 \pm 0,7$ & $25,55 \pm 1,6$ & $13,94 \pm 0,6$ & $1,84 \pm 0,2$ & $3,41 \pm 0,7$ \\
\hline \multirow{6}{*}{$\begin{array}{l}\text { Parede externa } \\
\text { do fruto }\end{array}$} & Verde-maduro & $58,29 \pm 2,1$ & $-9,31 \pm 0,8$ & $24,64 \pm 2,1$ & $-0,38 \pm 0,0$ & $0,14 \pm \quad 0,0$ \\
\hline & Verde-rosado & $54,89 \pm 5,2$ & $-4,76 \pm 2,7$ & $28,70 \pm 3,2$ & $-0,16 \pm 0,8$ & $0,03 \pm 0,0$ \\
\hline & Rosa-esverdeado & $52,66 \pm 4,4$ & $6,34 \pm 5,1$ & $30,91 \pm 1,3$ & $0,20 \pm 0,2$ & $0,06 \pm 0,1$ \\
\hline & Róseo & $42,65 \pm 2,3$ & $24,63 \pm 3,6$ & $25,33 \pm 2,2$ & $0,98 \pm 0,2$ & $0,98 \pm 0,3$ \\
\hline & Vermelho-claro & $39,99 \pm 3,4$ & $28,55 \pm 2,4$ & $24,97 \pm 2,8$ & $1,15 \pm 0,1$ & $1,34 \pm$ \\
\hline & Vermelho & $36,65 \pm 1,1$ & $27,16 \pm 2,5$ & $18,84 \pm 1,3$ & $1,44 \pm 0,1$ & $20,8 \pm 0,1$ \\
\hline \multirow{6}{*}{$\begin{array}{l}\text { Polpa } \\
\text { homogeneizada }\end{array}$} & Verde-maduro & $70,75 \pm 5,3$ & $-13,43 \pm 5,6$ & $38,43 \pm 4,1$ & $-0,34 \pm 0,1$ & $0,13 \pm 0,1$ \\
\hline & Verde-rosado & $68,15 \pm 8,2$ & $-1,65 \pm 3,3$ & $43,16 \pm 4,9$ & $-0,03 \pm 0,1$ & $0,01 \pm 0,0$ \\
\hline & Rosa-esverdeado & $62,12 \pm 2,8$ & $18,35 \pm 6,0$ & $41,38 \pm 2,2$ & $0,44 \pm 0,2$ & $0,21 \pm$ \\
\hline & Róseo & $50,17 \pm 4,4$ & $40,00 \pm 3,5$ & $33,80 \pm 4,4$ & $1,20 \pm 0,2$ & $1,48 \pm \quad 0,5$ \\
\hline & Vermelho-claro & $48,82 \pm 1,3$ & $42,65 \pm 3,5$ & $30,05 \pm 2,1$ & $1,42 \pm \quad 0,1$ & $2,02 \pm \quad 0,3$ \\
\hline & Vermelho & $44,27 \pm 5,2$ & $46,65 \pm 3,1$ & $26,75 \pm 1,4$ & $1,74 \pm 0,1$ & $3,05 \pm 0,3$ \\
\hline
\end{tabular}

Tabela 2. Valores dos componentes colorimétricos L*, a* e b* de frutos do híbrido 'RPT 1095' obtidos de diferentes estádios de maturação em três tipos de amostragem. Brasília, Embrapa Hortaliças, 2004.

\begin{tabular}{|c|c|c|c|c|c|c|}
\hline $\begin{array}{l}\text { Método de } \\
\text { amostragem }\end{array}$ & $\begin{array}{c}\text { Estádios de } \\
\text { maturação }\end{array}$ & $L^{*}$ & $a^{*}$ & $\mathbf{b}^{*}$ & $a^{*} / b^{*}$ & $\left(a^{*} / b^{*}\right)^{2}$ \\
\hline \multirow{6}{*}{$\begin{array}{l}\text { Parede interna } \\
\text { do fruto }\end{array}$} & Verde-maduro & $47,85 \pm 7,8$ & $-11,6 \pm 3,8$ & $30,48 \pm 7,5$ & $-0,38 \pm 0,0$ & $0,14 \pm 0,0$ \\
\hline & Verde-rosado & $46,56 \pm 2,8$ & $0,47 \pm 6,9$ & $26,03 \pm 4,6$ & $-0,01 \pm 0,3$ & $0,05 \pm 0,0$ \\
\hline & Rosa-esverdeado & $47,96 \pm 1,4$ & $-0,77 \pm 3,7$ & $23,60 \pm 2,5$ & $-0,04 \pm 0,2$ & $0,02 \pm 0,0$ \\
\hline & Róseo & $48,16 \pm 2,8$ & $2,70 \pm 6,9$ & $23,40 \pm 2,9$ & $0,11 \pm 0,3$ & $0,08 \pm 0,0$ \\
\hline & Vermelho-claro & $36,87 \pm 2,3$ & $24,4 \pm 4,4$ & $24,40 \pm 2,9$ & $0,99 \pm 0,1$ & $1,00 \pm 0,2$ \\
\hline & Vermelho & $31,93 \pm 1,2$ & $29,48 \pm 5,3$ & $20,48 \pm 4,7$ & $1,46 \pm 0,1$ & $2,13 \pm 0,4$ \\
\hline \multirow{6}{*}{$\begin{array}{l}\text { Parede externa } \\
\text { do fruto }\end{array}$} & Verde-maduro & $57,13 \pm 4,2$ & $-10,50 \pm 3,1$ & $32,73 \pm 1,3$ & $-0,33 \pm 0,1$ & $0,12 \pm 0,1$ \\
\hline & Verde-rosado & $54,37 \pm 1,7$ & $-6,33 \pm 2,5$ & $26,67 \pm 4,3$ & $-0,23 \pm 0,1$ & $0,06 \pm 0,0$ \\
\hline & Rosa-esverdeado & $50,40 \pm 3,3$ & $2,70 \pm 5,7$ & $26,30 \pm 4,2$ & $0,11 \pm 0,2$ & $0,05 \pm 0,1$ \\
\hline & Róseo & $44,37 \pm 5,7$ & $16,10 \pm \quad 1,4$ & $35,17 \pm 1,6$ & $0,62 \pm 0,6$ & $0,59 \pm 0,3$ \\
\hline & Vermelho-claro & $39,07 \pm 0,7$ & $24,23 \pm 6,4$ & $22,30 \pm 2,7$ & $1,07 \pm 0,3$ & $1,17 \pm 0,3$ \\
\hline & Vermelho & $36,78 \pm 0,5$ & $28,58 \pm 3,1$ & $20,28 \pm 1,2$ & $1,41 \pm 0,1$ & $1,98 \pm 0,2$ \\
\hline \multirow{6}{*}{$\begin{array}{l}\text { Polpa } \\
\text { homogeneizada }\end{array}$} & Verde-maduro & $70,70 \pm 1,2$ & $-12,55 \pm 5,9$ & $38,23 \pm 7,5$ & $-0,31 \pm 0,1$ & $0,11 \pm \quad 0,1$ \\
\hline & Verde-rosado & $73,37 \pm 2,7$ & $-3,40 \pm 2,7$ & $33,00 \pm 2,6$ & $-0,10 \pm 0,1$ & $0,02 \pm 0,0$ \\
\hline & Rosa-esverdeado & $68,73 \pm 1,4$ & $7,03 \pm 1,5$ & $31,93 \pm 4,5$ & $0,22 \pm 0,1$ & $0,06 \pm 0,0$ \\
\hline & Róseo & $61,33 \pm 6,3$ & $20,96 \pm 1,3$ & $28,73 \pm 2,1$ & $0,74 \pm 0,5$ & $0,68 \pm 0,6$ \\
\hline & Vermelho-claro & $50,53 \pm 4,4$ & $36,77 \pm 2,9$ & $29,66 \pm 1,7$ & $1,25 \pm 0,2$ & $1,57 \pm 0,4$ \\
\hline & Vermelho & $46,13 \pm 2,7$ & $42,70 \pm 4,9$ & $27,80 \pm 1,2$ & $1,53 \pm 0,1$ & $2,37 \pm 0,4$ \\
\hline
\end{tabular}

interna e externa, e na polpa dos frutos, apresentaram elevada correlação com os teores de licopeno analisados $\left(\mathrm{R}^{2}=0,81\right.$; 0,79 e 0,82 , respectivamente). Este resultado era de alguma forma esperado, já que o licopeno é um pigmento de coloração vermelha. Para os valores do componente $\mathrm{a}^{*}$ Arias et al. (2000) obtiveram valores de correlação $\left(\mathrm{R}^{2}\right)$ entre 0,82 (regressão linear) e 0,96 (regressão exponencial) associados aos teores de licopeno.

Em todos os estádios de maturação observou-se uma baixa correlação entre os valores de $b^{*}$ (grau da cor amarela do fruto) e os teores de licopeno nos frutos $\left(R^{2}=0,27\right.$ a 0,46$)$ (Tabela 4$)$. No entanto, maiores valores de $\mathrm{R}^{2}$ foram observados quando se correlacionou as relações $a^{*} / b^{*}$ e $\left(a^{*} / b^{*}\right)^{2}$ com as leituras feitas na superfície das paredes interna e externa dos frutos e também na polpa homogeneizada. Os valores das relações a*/b* e $\left(a^{*} / b^{*}\right)^{2}$ têm sido usados como indicadores do desenvolvimento da cor vermelha em frutos de tomate (D' SOUZA et al., 1992; ARIAS et al., 2000; LÓPEZ CAMELO; GÓMEZ, 2004). As 
Tabela 3. Valores dos componentes L', a* e b* de frutos do híbrido 'UG 096' obtidos de diferentes estádios de maturação em três tipos de amostragem. Brasília, Embrapa Hortaliças, 2004.

\begin{tabular}{|c|c|c|c|c|c|c|}
\hline $\begin{array}{l}\text { Método de } \\
\text { amostragem }\end{array}$ & $\begin{array}{c}\text { Estádios de } \\
\text { maturação }\end{array}$ & $L^{*}$ & $a^{*}$ & $\mathbf{b}^{*}$ & $a^{*} / b^{*}$ & $\left(a^{*} / b^{*}\right)^{2}$ \\
\hline \multirow{6}{*}{$\begin{array}{l}\text { Parede interna } \\
\text { do fruto }\end{array}$} & Verde-maduro & $43,88 \pm 2,5$ & $-11,73 \pm 1,5$ & $22,56 \pm 3,4$ & $-0,52 \pm 0,0$ & $0,28 \pm 0,0$ \\
\hline & Verde-rosado & $41,45 \pm 1,3$ & $5,30 \pm 1,9$ & $21,70 \pm 5,9$ & $0,26 \pm 0,2$ & $0,08 \pm 0,1$ \\
\hline & Rosa-esverdeado & $41,29 \pm 2,1$ & $14,46 \pm 4,6$ & $25,65 \pm 3,9$ & $0,57 \pm 0,2$ & $0,35 \pm 0,2$ \\
\hline & Róseo & $39,38 \pm 3,3$ & $27,71 \pm 5,8$ & $28,25 \pm 2,7$ & $0,99 \pm 0,2$ & $1,01 \pm 0,4$ \\
\hline & Vermelho-claro & $36,50 \pm 3,8$ & $28,08 \pm 3,7$ & $23,93 \pm 2,9$ & $1,18 \pm 0,2$ & $1,43 \pm 0,5$ \\
\hline & Vermelho & $34,38 \pm 0,9$ & $29,58 \pm 1,6$ & $20,33 \pm 0,8$ & $1,46 \pm 0,1$ & $2,13 \pm 0,3$ \\
\hline \multirow{6}{*}{$\begin{array}{l}\text { Parede externa } \\
\text { do fruto }\end{array}$} & Verde-maduro & $48,30 \pm 3,2$ & $-13,26 \pm 1,3$ & $24,68 \pm 0,8$ & $-0,54 \pm 0,0$ & $0,28 \pm 0,0$ \\
\hline & Verde-rosado & $44,73 \pm 0,2$ & $8,23 \pm 2,0$ & $27,20 \pm 0,9$ & $0,30 \pm 0,1$ & $0,01 \pm 0,0$ \\
\hline & Rosa-esverdeado & $41,76 \pm 1,9$ & $15,96 \pm 5,4$ & $25,95 \pm 2,0$ & $0,62 \pm 0,2$ & $0,41 \pm 0,2$ \\
\hline & Róseo & $43,58 \pm 2,1$ & $25,08 \pm 1,2$ & $28,58 \pm 2,3$ & $0,88 \pm 0,1$ & $0,79 \pm 0,2$ \\
\hline & Vermelho-claro & $38,73 \pm 1,2$ & $28,65 \pm 1,2$ & $25,10 \pm 2,5$ & $1,15 \pm 0,1$ & $1,33 \pm 0,2$ \\
\hline & Vermelho & $37,01 \pm 2,4$ & $29,13 \pm 2,3$ & $22,46 \pm 1,9$ & $1,30 \pm 0,1$ & $1,70 \pm 0,3$ \\
\hline \multirow{6}{*}{$\begin{array}{l}\text { Polpa } \\
\text { homogeneizada }\end{array}$} & Verde-maduro & $66,55 \pm 6,3$ & $-17,33 \pm 1,9$ & $40,98 \pm 4,5$ & $-0,42 \pm 0,0$ & $0,18 \pm 0,0$ \\
\hline & Verde-rosado & $64,40 \pm 2,6$ & $9,40 \pm 2,1$ & $39,85 \pm 2,2$ & $0,24 \pm 0,1$ & $0,06 \pm 0,0$ \\
\hline & Rosa-esverdeado & $61,45 \pm 1,0$ & $20,80 \pm 3,6$ & $33,23 \pm 0,6$ & $0,63 \pm 0,1$ & $0,40 \pm 0,2$ \\
\hline & Róseo & $50,58 \pm 2,4$ & $36,55 \pm 2,9$ & $34,50 \pm 2,4$ & $1,06 \pm 0,0$ & $1,12 \pm \quad 0,1$ \\
\hline & Vermelho-claro & $52,00 \pm 2,6$ & $37,85 \pm 2,9$ & $33,82 \pm 0,5$ & $1,12 \pm 0,1$ & $1,26 \pm 0,2$ \\
\hline & Vermelho & $42,35 \pm 4,4$ & $37,03 \pm 3,7$ & $25,43 \pm 2,6$ & $1,46 \pm 0,1$ & $2,13 \pm 0,3$ \\
\hline
\end{tabular}

Tabela 4. Coeficientes de determinação da regressão linear simples $\left(\mathrm{R}^{2}\right)$ entre teor de licopeno e o componente acromático $L^{*}$ (grau de brilho) e entre o teor de licopeno e os componentes cromáticos $\mathrm{a}^{*}\left(+\mathrm{a}^{*}\right.$ : grau da cor vermelha do fruto; $-\mathrm{a}^{*}$ : grau da cor verde) e $\mathrm{b}^{*}\left(+\mathrm{b}^{*}\right.$ : grau da cor amarela; - - ${ }^{*}$ : grau da cor azul) bem como com as relações $a^{*} / b^{*}$ e $\left(a^{*} / b^{*}\right)^{2}$. Leituras colorimétricas foram obtidas a partir de amostragem da parede externa dos frutos (cor externa), parede interna dos frutos (cor interna) e polpa homogeneizada (polpa) de frutos de tomateiro. Brasília, Embrapa Hortaliças, 2004.

\begin{tabular}{cccc}
\hline $\begin{array}{c}\text { Valores de } \\
\text { cromaticidade }\end{array}$ & $\mathbf{R}^{2}$ Cor interna & $\mathbf{R}^{2}$ Cor externa & $\mathbf{R}^{2}$ Cor da polpa \\
\hline $\mathrm{L}^{*}$ & $0,63 \mathrm{~s}$ & $0,67 \mathrm{~s}$ & $0,82 \mathrm{~s}$ \\
$\mathrm{a}^{*}$ & $0,81 \mathrm{~s}$ & $0,79 \mathrm{~s}$ & $0,82 \mathrm{~s}$ \\
$\mathrm{~b}^{*}$ & $0,36 \mathrm{~s}$ & $0,27 \mathrm{~s}$ & $0,46 \mathrm{~s}$ \\
$\mathrm{a}^{*} / \mathrm{b}^{*}$ & $0,90 \mathrm{~s}$ & $0,88 \mathrm{~s}$ & $0,90 \mathrm{~s}$ \\
$\left(\mathrm{a}^{*} / \mathrm{b}^{*}\right)^{2}$ & $0,86 \mathrm{~s}$ & $0,89 \mathrm{~s}$ & $0,91 \mathrm{~s}$ \\
\hline
\end{tabular}

s Significante para P menor ou igual a 0,05

relações $a^{*} / b^{*}$ e $\left(a^{*} / b^{*}\right)^{2}$ têm-se mostrado bons parâmetros quando se procura ajustar uma regressão linear envolvendo os diferentes estádios de maturação de tomate.

Observou-se uma variação entre 0,88 a 0,90 para $a^{*} / b^{*}$ e de 0,86 a 0,91 para $\left(a^{*} / b^{*}\right)^{2}$, sendo que os valores obtidos com a polpa homogeneizada foram, consistentemente, os mais elevados (Tabela 4). Os gráficos da regressão linear do teor de licopeno $(\mu \mathrm{g} / \mathrm{g})$ em frutos de tomateiro versus os valores de cromaticidade das relações $\mathrm{a}^{*} / \mathrm{b}^{*} \mathrm{e}(\mathrm{a} * /$ $\left.b^{*}\right)^{2}$ obtidos a partir de amostragem das paredes interna externa e interna e da polpa homogeneizada de frutos de tomateiro encontram-se na Figura 1. As regressões lineares entre as relações a*/ $\mathrm{b}^{*}$ e $\left(\mathrm{a}^{*} / \mathrm{b}^{*}\right)^{2}$ com a concentração de licopeno na polpa apresentaram elevada correlação $\left(\mathrm{R}^{2}=0.90\right.$ e $\mathrm{R}^{2}=0.91$, respectivamente). Consequentemente, as estimativas dos valores de licopeno poderão ser feitas utilizando-se as seguintes equações:

(1)

$(\mu \mathrm{g} / \mathrm{g}$ de licopeno $)=33,92\left(\mathrm{a}^{*} / \mathrm{b}^{*}\right)^{2}+10,633$ ou (2)

$(\mu \mathrm{g} / \mathrm{g}$ de licopeno $)=45,912\left(\mathrm{a}^{*} / \mathrm{b}^{*}\right)+12,863$

Devido à conveniência e a facilidade no uso de medidas de cor via colorímetro, vários estudos têm investigado a correlação entre o teor de pigmentos e a coloração de diferentes hortaliças, tais como pimentão do tipo "paprika" (RAMAKRISHNAN; FRANCIS, 1973), pimenta vermelha (REEVES, 1987), folhas desidratadas de salsa (BERSET; CANIAUX, 1983), batata-doce (AMENY; WILSON, 1997; TAKAHATA et al., 1993), cenoura (LING et al., 1996; CHEN; TANG, 1998), melancia (PERKINS-VEAZIE et al., 2001) e em frutos do tomateiro (D'SOUZA et al. 1992; ARIAS et al. 2000).

Os resultados obtidos no presente trabalho indicam que a medida da coloração da polpa homogeneizada pode ser considerada como uma das melhores alternativas para se estimar indiretamente a concentração de licopeno por meio da cromaticidade em frutos do tomateiro. A utilização de polpa homogeneizada para medidas de cromaticidade havia sido anteriormente apenas sugerida por PERKINS-VEAZIE et al. (2000), em melancia, como uma opção metodológica, mas nunca efetivamente utilizada nesta e em outras espécies vegetais. Desta forma, o estudo reportado aqui é a primeira demonstração da eficiência relativa desta estratégia de amostragem colorimétrica. As prováveis causas da preferência pelas medidas 


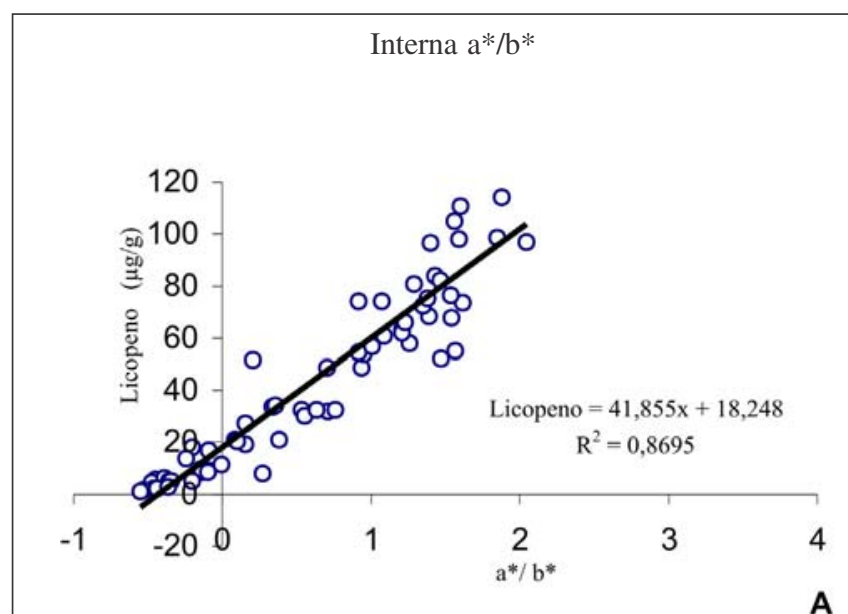

Externa $\mathrm{a}^{*} / \mathrm{b}^{*}$

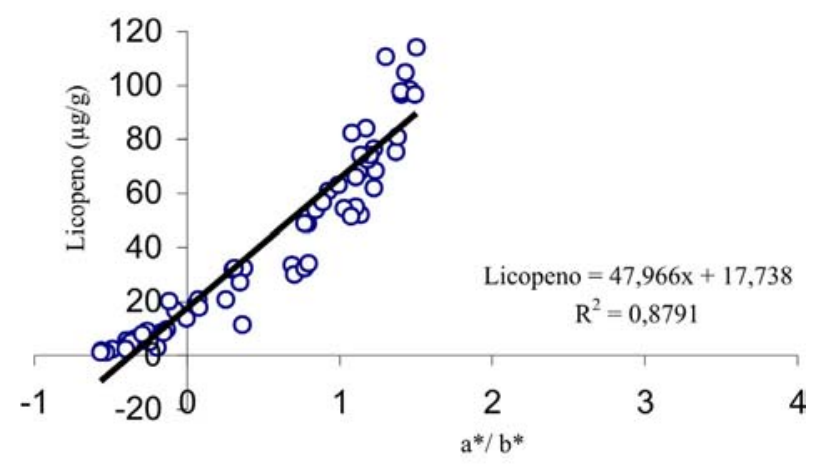

C

Polpa $a * / b *$
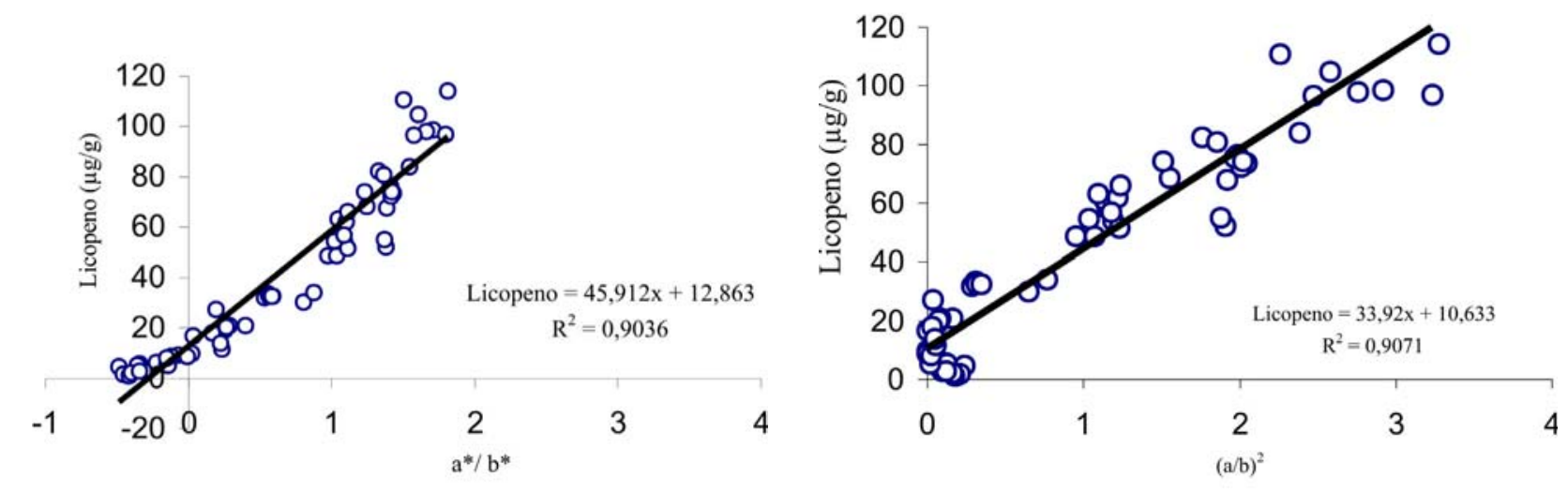

E

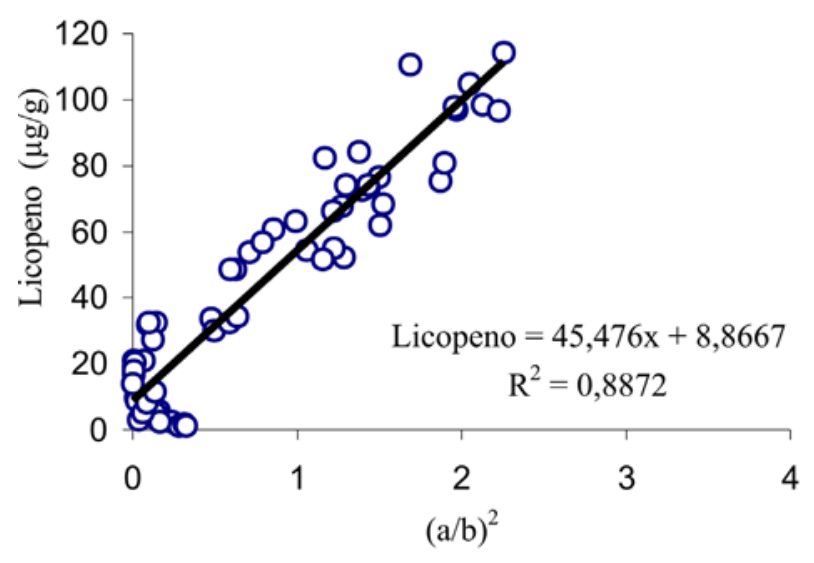

Polpa $\left(\mathrm{a}^{* / \mathrm{b}}\right)^{2}$

Figura 1. Gráficos de regressão linear do teor de licopeno $(\mu \mathrm{g} / \mathrm{g})$ em frutos de tomateiro versus os valores de cromaticidade das relações $\mathrm{a} * / \mathrm{b}^{*} \mathrm{e}\left(\mathrm{a} * / \mathrm{b}^{*}\right)^{2}$ obtidos a partir de amostragem da parede interna dos frutos (interna; gráficos A \& B), parede externa dos frutos (externa; gráficos C \& D) e polpa homogeneizada (polpa; gráficos E \& F) de frutos de tomateiro. Brasília, Embrapa Hortaliças, 2004.

colorimétricas de cor na superfície de tomates em detrimento da utilização de leituras da polpa do fruto homogeneizada observadas na literatura (D'SOUZA et al. 1992; ARIAS et al. 2000) não são conhecidas. Esta preferência estaria pos- sivelmente relacionada com alguns aspectos negativos da amostragem usando a polpa do fruto homogeneizada tais 
como: (1) o procedimento ser mais demorado e (2) a amostragem ser necessariamente destrutiva. No entanto, no caso do melhoramento genético do tomateiro, estes dois aspectos não apresentam relevância significativa. Como indicado por resultados da presente pesquisa, a relativa demora do procedimento para obter polpa homogeneizada pode ser compensada pelo incremento na precisão. $\mathrm{O}$ fato de a análise ser destrutiva, não oferece, no caso do tomateiro, maiores limitações uma vez que esta hortaliça produz dezenas de frutos por planta, permitindo que apenas um subgrupo seja amostrado. Além disso, a amostra processada desta forma está pronta para uma série de análises de parâmetros de qualidade tais como: teor de sólidos solúveis e viscosidade.

Neste contexto, o método descrito no presente trabalho apresenta uma série de vantagens além da precisão, tais como praticidade, economia de reagentes e baixo custo de implementação constituindo-se, portanto, em uma ferramenta opcional no processo de seleção de genótipos superiores para teores de licopeno em programas de melhoramento genético de tomateiro. Além disso, a estratégia de amostragem utilizando homogeneização da polpa permite que sejam feitas poucas e mais consistentes leituras da cromaticidade, evitando-se a necessidade de obter várias leituras do mesmo fruto a fim de que os coeficientes de variação, normalmente elevados, sejam reduzidos (D'SOUZA et al. 1992; ARIAS et al. 2000). Desta forma, a estratégia ora descrita irá facilitar a constituição de amostras com maior representativilidade do genótipo/acesso a ser avaliado, permitindo estimar indiretamente e com maior segurança, o teor de licopeno utilizando-se de um colorímetro.

\section{LITERATURA CITADA}

AMENY, M.; WILSON, P. Relationship between Hunter color values and beta-carotene contents in white fleshed African sweet potatoes (Ipomoea batatas Lam). Journal of Science Food Agriculture, v.73, n.3, p.301-306, 1997.

ARIAS, R.; LEE, T.C.; LOGENDRA L.; JANES,

$\mathrm{H}$. Correlation of lycopene measured by HPLC with the $\mathrm{L}^{*}, \mathrm{a}^{*}, \mathrm{~b}^{*}$ color readings of a hidroponic tomato and the relationship of maturity with color and lycopene content. Journal of A gricultural and Food Chemistry, v.48, p.1697-1702, 2000.

BERSET, C.; CANIAUX, P. Relationship between color evaluation and chlorophyllian pigment content in dried parsley leaves. Journal of Food Science, v.48, p.1854-1857, 1983.

BOILEAU, T.W.; LIAO, Z.M.; KIM, S.; LEMESHOW, S.; ERDMAN, J.W.; CLINTON, S.K. Prostate carcinogenesis in $N$-methyl- $N$ nitrosourea (NMU)-testosterone-treated rats fed with tomato powder, lycopene, or energyrestricted diets. Journal of the National Cancer Institute, v. 95, n. 21, p.1578-1586, 2003.

CHEN, B.H.; TANG, Y.C. Processing and stability of carotene powder from carrot pulp waste. Journal of Agricultural Food Chemistry, v.46, p.2312-2318, 1998.

CRAMER, D.W.; KUPER, H.; HARLOW, B.L.; TITUS-ERNSTOFF, L. Carotenoids, antioxidants and ovarian cancer risk in pre- and postmenopausal women. International Journal of Cancer, v.94, n.1, p.128-134, 2001.

D'SOUZA, M.C.; SINGHA, S.; MORRIS I. Lycopene concentration of tomato fruit can be estimated from chromaticity values. HortScience, v.27, n.5, p.465-466, 1992.

LIME, B.J.; GRIFFITHS, F.P.; O’CONNOR, R.T.; HEINZELMANN, D.C.; McCALL, E.R. Spectrophotometer methods for determining pigmentation - beta-carotene and lycopene - in ruby red grapefruit. Agricultural and Food Chemistry, v.5, p.941-944, 1957.

LING, P.; RUZHITSKY, V.; KAPANIDIS, A.; LEE,

T.C. Correlation between color machine vision and colorimeter for food applications. Chemical markers for processed and stored foods. ACS Symposium Service No. 631, 1996. p.253-278.
LÓPEZCAMELO, A.F.; GÓMEZ, P.A. Comparison of color indexes for tomato ripening. Horticultura Brasileira, Brasília, v.22, p.534-537, 2004.

MORETTI, C.L.; SARGENT, S.A.; HUBER, D.J.; CALBO, A.G.; PUSCHMANN, R. Chemical composition and physical properties of pericarp, locule, and placental tissues of tomatoes with internal bruising. Journal of the American Society for Horticultural Science, v.123, n.4, p.656-660, 1998.

NGUYEN, M.L.; SCHWARTZ, S.J. Lycopene: chemical and biological properties. Food Technology, v.53, n.2, p.38-45. 1999.

PERKINS-VEAZIE, P.; COLLINS, J.K.; PAIR, S.D.; ROBERTS, W. Lycopene content differs among red-fleshed watermelon cultivars. Journal of Science Food and Agriculture v.81, p.983-987, 2001.

RAMAKRISHNAN, T.; FRANCIS, F. Color and carotene changes in heated paprika. Journal of Food Science, v.39, n.1, p.25-28, 1973.

RAO, A.V. Lycopene, tomatoes, and the prevention of coronary heart disease. Experimental Biology and Medicine, v.227, p.908-913, 2002. RAO, A.V.; AGAWAL, S. Role of antioxidant lycopene in cancer and heart disease. Journal of the American College of Nutrition, v.19, n.5, p.563-569, 2000.

RAO, A.V.; WASEEM, Z.; AGAWAL, S. Lycopene content of tomatoes and tomato products and their contribution to dietary lycopene. Food Research International, v.31, n.10, p.737-741, 1998.

REEVES, M. Re-evaluation of Capsicum color data. Journal of Food Science, v.52, p.1047-1049, 1987. RODRIGUEZ-AMAYA, D. A Guide to Carotenoids Analysis in Food. Washington: International Life Sciences Institute Press, 2001. 64p.

SAS INSTITUTE. SAS User's Guide. SAS Institute, Cary, North Caroline, USA, 1982.

TAKAHATA, Y.; NODA, T.; NAGATA, T. HPLC determination of beta-carotene content of sweet potato cultivars and its relationship with color values. Japanese Journal of Breeding, v.43, n.3, p.421-427, 1993.

THE CALIFORNIA TOMATO BOARD. Ripening Stages for Tomatoes. USDA Visual Aid, 1975.

UMIEL, N.; GABELMAN, W.H. Inheritance of root colour and carotene synthesis in carrot, Daucus carota L. orange vs. red. Journal of the American Society for Horticultural Science, v. 97, n.4, p. 453-460, 1971. 